\title{
UNIVERSITYOF
}

FORWARD

THINKING

WESTMINSTER用

WestminsterResearch

http://www.westminster.ac.uk/westminsterresearch

The measurement, mathematical and logical modeling, and agentbased simulation of carbon intangible assets with embedded strategies

Zeng, Y., Li, Shuliang and Deng, Liangru

This is a copy of the author's accepted version of a paper subsequently to be published in the proceedings of the 2nd International Conference on Knowledge Engineering and Applications (ICKEA2017), Imperial College London, UK, 21 to 23 Oct 2017.

It is available online at:

https://doi.org/10.1109/ICKEA.2017.8169905

(C) 2017 IEEE . Personal use of this material is permitted. Permission from IEEE must be obtained for all other uses, in any current or future media, including reprinting/republishing this material for advertising or promotional purposes, creating new collective works, for resale or redistribution to servers or lists, or reuse of any copyrighted component of this work in other works.

The WestminsterResearch online digital archive at the University of Westminster aims to make the research output of the University available to a wider audience. Copyright and Moral Rights remain with the authors and/or copyright owners.

Whilst further distribution of specific materials from within this archive is forbidden, you may freely distribute the URL of WestminsterResearch: ((http://westminsterresearch.wmin.ac.uk/)).

In case of abuse or copyright appearing without permission e-mail repository@westminster.ac.uk 


\section{The Measurement, Mathematical and Logical Modeling, and Agent-based Simulation of Carbon Intangible Assets with Embedded Strategies}

\author{
Yin Zeng ${ }^{1}$, Shuliang $\mathrm{Li}^{1,2}$ \\ ${ }^{1}$ School of Economics \& Management, \\ Southwest Jiaotong University \\ Chengdu, China \\ ${ }^{2}$ Westminster Business School, University of \\ Westminster \\ London, United Kingdom \\ e-mail: zengyin@live.cn, s.li@westminster.ac.uk
}

\author{
Liangru Deng \\ Chengdu Seqdata Technology Co., Ltd \\ Chengdu, China \\ e-mail: liangrudeng@163.com
}

\begin{abstract}
A measurement model for quantifying the intangible value of enterprise carbon management is proposed and established in this paper. We devise and formulate embedded management strategies of the assets for automobile enterprises, and develop a software agent-based system for simulating the evolution of the assets of automobile enterprises under three different scenarios. The results show that our measurementbased simulation paradigm and method are effective in measuring and analyzing the assets and their evolutionary characteristics and laws.
\end{abstract}

Keywords-Carbon intangible asset; management strategy; software agent; mathematical and logical modeling; simulation

\section{INTRODUCTION}

This article is based on the carbon emission rights as intangible assets proposed by Berkhout and Smith [1], the accounting treatment of carbon intangible assets by Gunther [2] and Lewandowski [3]. In addition to the carbon emission rights, this paper also examines the patent technology of emission reduction and energy saving, low carbon brand effect, advanced carbon management methods, the measurement principle of other carbon intangible assets, etc. In addition, intangible assets are essential to enterprise development in the new economy, and therefore, the mathematical model of measurement and analysis needs to be further established. In view of the performance of carbon responsibilities enterprises need to evaluate effectively and predict the value contribution of carbon intangible assets [47]. The key point is to integrate the intangible asset theory with the carbon emission reduction regulations, and to propose the interactive model that can depict the assets and effectively reflect the carbon management decisions, and thus help enterprises avoid the risk of carbon decisionmaking and improve the precision of low carbon management for enterprises.

\section{Value Measurement Model of Enterprise CARBON INTANGIBLE ASSETS}

On the basis of Zeng and Li [8]'s work on enterprise carbon asset multi-agent simulation, this paper further defines and quantifies the intangible value created by enterprises carbon management, and probes into the laws and characteristics of interactive evolution across the assets.

\section{A. Low carbon Technology}

Through technological research and development, enterprises can acquire low carbon technologies with carbon emission reduction capabilities, which can be converted into various values, such as improvements on efficiency and technology transfer. The carbon emission reduction technology has the value attribute of carbon intangible assets [9], and the created value reflects the comprehensive competitiveness of the low carbon technologies of enterprises.

$$
V_{t}=\sum_{i=1}^{n}\left(M_{i}-C_{i}\right) \cdot Y \cdot\left(p-\beta \ln \left(1-\frac{Q}{\sum_{j} E_{j}\left(1-r_{j}\right)}\right)\right)
$$

where $M_{i}$ stands for the industry carbon emission baseline of the technology; $C_{i}$ denotes the comprehensive carbon emission level of the technology; $Y$ is the sales in the research period; $V_{t}$ is the overall carbon emission reduction value of an enterprise's low carbon technologies; $n$ stands for the number of enterprise low carbon technologies $; \square$ is the relevant parameter; $r_{j}$ shows the distance between the carbon intensity of the enterprise and the carbon emission rate corresponding to the average carbon intensity of the industry; $E_{j}$ represents enterprise carbon emissions; and $Q$ is the total carbon quota of the industry.

\section{B. Carbon Sinks}

Under the Kyoto Protocol, the verified carbon sinks developed by enterprise has the value to offset carbon emissions or trade. The development of carbon sinks is based on the actual needs of carbon emission reduction of enterprises, or the activity of developing carbon sinks with greater carbon transaction value through the use of market information. For example, enterprises develop carbon sequestration projects. The theoretical model of calculating carbon reserves is as follows [10]: 
Zeng, Yin \& Li, Shuliang \& Deng, Liangru. "The measurement, mathematical and logical modeling, and agent-based simulation of carbon intangible assets with embedded strategies". Proceedings of the 2nd International Conference on Knowledge Engineering and Applications (ICKEA2017), pp.70-74. 21 st to 23rd Oct 2017, Imperial College London, UK. IEEE. ISBN 978-1-5386-2149-3 \& ISBN 978-1-5386-2148-6/17

$$
V(t)=\int_{0}^{t}(1-W-L C) q^{\prime}(t) d t
$$

where $V(t)$ stands for the actual carbon accumulation of an individual tree species of year $t ; q^{\prime}(t)$ is the derivative of $q(t)$ representing the natural carbon accumulation of an individual tree species in year $t ; W$ denotes the loss ratio of carbon sequestration; and $L C$ strands for the deforestation or reduction rate.

Based on its carbon sink strategy, an enterprise can decide on the scale that needs to be developed, in order to get the corresponding carbon sinks $C(t)$. The value of carbon sinks, $V_{c}$, can be obtained by taking away the carbon sink development and maintenance costs.

$$
\begin{aligned}
V_{c} & =C(t) \cdot p-C_{c} \\
& =p \cdot N \cdot M \cdot \frac{D}{R_{t}} \cdot \frac{C_{i}}{T_{C}} \cdot(1-W-L C) \cdot \mu(1-\exp (-k t))^{b}-C_{c}
\end{aligned}
$$

where $N$ denotes the carbon sink development scale or area; $M$ strands for the planting density per unit area; $D$ stands for the stem density of the carbon sink tree species; $R_{t}$ denotes the biomass of tree trunk occupies the proportion of the biomass of the arbor layer; $C_{i}$ is the carbon content of the carbon sink tree; $T_{c}$ is the proportion of carbon in molecular weight of $\mathrm{CO} 2 ; \square, k$ and $b$ are the corresponding parameters of the carbon sink tree, respectively; $C_{c}$ stands for the total cost of developing carbon sinks; and $p$ represents the carbon trading market price.

\section{Clean Production Level}

Clean production audit is the legal requirement of national energy conservation and emission reduction. It is also the inevitable choice of low carbon development of enterprises. Each enterprise has its corresponding production functions, and the value of the acquired carbon assets can be obtained by the enterprise implementation of the audit. By using the FEEM-RICE model of Bosetti [11] to introduce the conversion coefficient of carbon intangible assets, the value model of carbon intangible assets of clean production level is given below:

$$
V_{p}=A \cdot L^{\alpha} \cdot K^{\lambda} \cdot \frac{\sum_{i=1}^{n}\left(e^{i}-e\right) \sum_{j=1}^{\infty}(-1)^{j-1} \cdot \Delta a_{i}^{j}+\sum_{i=1}^{n} \Delta e^{i} \cdot a_{i}+\sum_{i=1}^{n} \Delta e^{i}\left(a_{i}-1\right) \sum_{j=2}^{\infty}(-1)^{j-1} \cdot \Delta a_{i}^{j-1}+\Delta e_{r}}{\sum_{i=1}^{n} Y_{i} \cdot e^{i}}
$$

In Equation (4), $V_{p}$ denotes the carbon intangible asset value of clean production level; $A$ is the comprehensive technical level of production; $L$ stands for the factor of labor force; $K$ is the input element of the physical capital; $D$ and $D$ stands for elasticity of labor output, and output elasticity of the physical capital, respectively; $E_{c}$ denotes an value conversion coefficient of carbon intangible assets, represents the results of carbon emission reduction in the production and operation of enterprises, and can be described by the carbon emission reduction rate per unit product; $Y_{i}$ is the sales of low carbon technology $i$; $e^{i}$ stands for the carbon emission intensity or carbon emission factor of low carbon technology $i ; \Delta e^{i}$ is the variable quantity of carbon emission intensity or carbon emission factor of low carbon technology $i ; \quad a_{\mathrm{i}}=Y_{i} / Y$ stands for sales volume with $i$ low carbon technology accounted for the total sales volume; and $\Delta a_{i}$ is an variable quantity compared to the reference time.

\section{Carbon Value Chain System}

On the basis of carbon value chain system, the enterprise can establish long-term cooperation to reduce emissions with retailers and suppliers, and improve the profits through low carbon products and thus realize Pareto improvements on emission reduction and value chain optimization. For instance, enterprises need to carry out material circulation or information flow in all aspects of business activities. Adopting more efficient circulation system can bring greater carbon emission reduction value. In order to facilitate the analysis, this paper will compare the low carbon products and traditional products developed by enterprises in improving of the carbon value chain system. And the created carbon profit space $V_{l}$ is analyzed using the following formula:

$$
V_{l}=\left\{p_{c}-p_{t}-p\left[(1-R) e-e_{q}\right]\right\} \cdot D(R)-C(R)
$$

where $p_{c}$ is the market price of low carbon products; $p_{t}$ is the market price of traditional products; $e_{q}$ is the allocation of carbon quotas from the government; $D(R)$ is the demand function of enterprise low carbon products; and $C(R)$ represents the cost reduction function of the completed carbon emission reduction rate $R$ of an enterprise.

\section{E. Carbon footprint (carbon disclosure)}

In the literature, scholars are mainly concerned with the correlation carbon disclosure for the enterprise value [12]. Through regression analysis, we can find out the factors that are relevant to the value and strengths of an enterprise. This lays a theoretic foundation to measure the intangible value of enterprise carbon disclosure:

$$
V_{d}=\frac{T}{E}=\frac{\sum_{k}\left(p_{v}^{k}-c_{v}^{k}\right) \cdot\left[Y_{0}^{k}+\delta\left(E_{0}-E\right)\right]}{\left(E_{0}+\sigma\right) \exp (\gamma t)+\sigma}
$$

In Equation (6), $V_{d}$ is the value contribution of carbon disclosure (carbon footprint); $T$ stands for the gross profit of enterprise products; $p_{v}^{k}$ is the price of product $k$; $c_{v}^{k}$ represents the cost of product $k ; Y_{k}$ denotes the sales of product $k ; E_{0}$ is the carbon emission of the base period; $\sigma$ represents the concomitant variable of enterprise carbon emissions, and reflects the increase and decrease of enterprise production scale; $\gamma$ stands for the attenuation coefficient of enterprise carbon emissions,; $t$ is the length of time relative to base time, calculated by year; $Y_{0}^{k}$ is a constant, representing the sales of product $k$ in base period; and $\delta$ is the influence coefficient of carbon emissions to product sales.

\section{F. Low Carbon Brand}

From the perspective of enterprises, brand value is to create extra profits. In this paper, enterprise brand value is 
Zeng, Yin \& Li, Shuliang \& Deng, Liangru. "The measurement, mathematical and logical modeling, and agent-based simulation of carbon intangible assets with embedded strategies". Proceedings of the 2nd International Conference on Knowledge Engineering and Applications (ICKEA2017), pp.70-74. 21 st to 23rd Oct 2017, Imperial College London, UK. IEEE. ISBN 978-1-5386-2149-3 \& ISBN 978-1-5386-2148-6/17

calculated from the brand advantage of an enterprise. The low carbon brand extension [13] is also considered. Hence, the value of the carbon intangible assets of low carbon brand $V_{b}$ is obtained through the following formula.

$$
V_{b}=\frac{(C P-C A P) \cdot Y}{C R} \cdot\left[\left(\omega_{1} \omega_{2} \omega_{3} \omega_{4} \omega_{5}\right) \cdot\left(\begin{array}{l}
m_{0}^{1} \cdot \exp \left(\tau_{1} t\right) \\
m_{0}^{2} \cdot \exp \left(\tau_{2} t\right) \\
m_{0}^{3} \cdot \exp \left(\tau_{3} t\right) \\
m_{0}^{4} \cdot \exp \left(\tau_{4} t\right) \\
m_{0}^{5} \cdot \exp \left(\tau_{5} t\right)
\end{array}\right)\right]
$$

where $C P$ stands for enterprise unit product profit; $C A P$ stands for average unit product profit of an industry; $C R$ is the average profit margin of industry unit product; $\omega_{i}$ represents the weight coefficient; $m_{0}^{i}$ denotes the initial value; $\tau_{i}$ stands for the time constant of the dynamic elongation of the $i$ th carbon intangible asset; and $t$ is the time span compared with baseline and is calculated in year.

\section{LOW CARBON MANAGEMENT STRATEGY DESIGN}

Carbon intangible assets are gradually formed and evolved under the complex management decisions of enterprises. In this process, there are interactive behaviors across carbon intangible assets which will be promoted, influenced or weakened. This paper provides a structural description [14] of the interactive mechanism of carbon intangible asset software agent, Agent, based on the value measurement model of the assets. The structure is presented below.

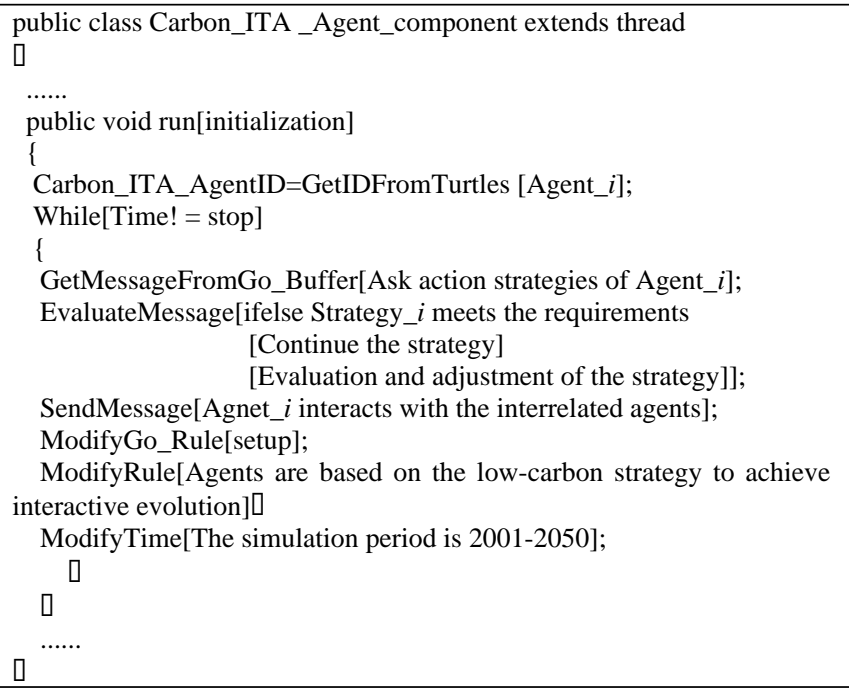

\section{The Simulation System}

\section{A. Model Specification}

This study adopts the Netlogo platform to build a simulation system, and fully implants the value contribution model of carbon intangible assets. On the basis of the interactive mechanism of the assets, the evolution results and trends of the assets are revealed and demonstrated in the implementation of an carbon management strategy, and hence provides low carbon decision support for managers. As shown in Fig. 1, diagram (1) is the simulation control interface; diagram (2) is the programming interface; (3) and (4) are the $3 d$ dynamic display interfaces of the simulation.

$$
\text { i: }
$$

Figure 1. Netlogo model simulation interface of the evolution interaction of enterprise carbon intangible assets

\section{B. Scenario Design}

In order to analyze the development of carbon intangible asset as Agents from different perspectives, and to explore how they influence each other, this paper takes the automobile enterprises as simulation samples and sets the enterprise low carbon development into three initial scenarios: the inferior situation, medium situation and advantaged situation. The three scenarios distinguishing the enterprise state of low carbon development in reality require complex comparisons. For the purpose of simplicity, the enterprise comprehensive carbon emission level of low carbon technology is compared to the industry benchmark as the criterion to identify the simulation situations. In addition, in order to make the simulation results more comparable, the equal initial production and operation scale of the enterprise in three scenarios is set. The initial value of state variables is mainly derived from the enterprise research and literature. Some parameters need to be adjusted during the process of simulation, in order to coordinate with the development strategy of the carbon intangible asset. The data is listed Table 1 .

\section{SimUlation RESUlts AND DiscUSSIONS}

The interaction evolution of the carbon intangible asset Agent is based on the corresponding decision variables. For example, the carbon intensity of each model in the simulation is calculated as follows:

$$
e_{i}(t)=\frac{e_{i}(t-1)}{1+k_{Y}}-\frac{a_{e}}{k_{e}+\exp \left(b_{e}-\frac{c_{e}}{t}\right)}
$$

In the formula, $e_{i}$ stands for the carbon intensity of the $i$ mode; $k_{Y}$ is the accommodation coefficient of sales volume $D$ When the sale rises, the coefficient is positive; when it decrease, the coefficient is negative $\left[k_{e}, a_{e}, b_{e}\right.$ and $c_{e}$ are corresponding parameters. Other decision variables will be expanded in detail in subsequent studies.

The simulation started from 2001, and ran for 50 years. Through the simulation experiment, enterprise carbon intangible assets present their own overall evolution process, and shows distinct differences in each scenario. This reflects the development characteristics of enterprise carbon intangible assets under the corresponding management 
Zeng, Yin \& Li, Shuliang \& Deng, Liangru. "The measurement, mathematical and logical modeling, and agent-based simulation of carbon intangible assets with embedded strategies". Proceedings of the 2nd International Conference on Knowledge Engineering and Applications (ICKEA2017), pp.70-74. 21 st to 23rd Oct 2017, Imperial College London, UK. IEEE. ISBN 978-1-5386-2149-3 \& ISBN 978-1-5386-2148-6/17

strategies. In addition, the influence factors leading to the differences in the evolution of the assets as a whole or between individuals can be found by comparison. The results indicates that, as long as an enterprise practices low carbon development path, the value contribution of its carbon intangible assets will emerge to various degrees, no matter in which scenario.

TABLE I. The INITIAL VALUE OF AUTOMOBILE ENTERPRISE IN THREE SCENARIOS

\begin{tabular}{|c|c|c|c|c|}
\hline variable & unit & $\begin{array}{c}\text { inferior } \\
\text { situation }\end{array}$ & $\begin{array}{l}\text { medium } \\
\text { situation }\end{array}$ & $\begin{array}{l}\text { advantage } \\
\text { d situation }\end{array}$ \\
\hline$M$ & $10,000 t$. & \multicolumn{3}{|c|}{50} \\
\hline$n$ & $\begin{array}{l}\text { Low carbon } \\
\text { vehicles }\end{array}$ & 0 & 3 & 8 \\
\hline$C$ & $10,000 \mathrm{t}$. & 70 & 48 & 36 \\
\hline$E$ & $10,000 t$. & 330 & 245 & 180 \\
\hline$R$ & - & 0.003 & 0.01 & 0.02 \\
\hline$Y$ & 10,000 unit & 120 & 160 & 220 \\
\hline$P$ & $€ / \mathrm{tCO}_{2}$ & 11.6 & 11.6 & 11.6 \\
\hline$N$ & ha. & 0 & 50 & 200 \\
\hline$W$ & - & $2.3 \%$ & $2.3 \%$ & $2.3 \%$ \\
\hline$L C$ & - & $1.8 \%$ & $1.8 \%$ & $1.8 \%$ \\
\hline$e$ & $\mathrm{tCO}_{2}$ /unit & 1.5 & 1.16 & 0.97 \\
\hline$K$ & 100 million $€$ & 250 & 330 & 450 \\
\hline$A$ & $*$ & 3600 & 5100 & 6800 \\
\hline$L$ & 10,000 people & 3.2 & 3.0 & 2.6 \\
\hline$p_{c}$ & $€ /$ unit & 22000 & 35000 & 63000 \\
\hline$p_{t}$ & $€ /$ unit & 14000 & 23000 & 32000 \\
\hline$C q$ & $10,000 \mathrm{t}$. & 260 & 240 & 180 \\
\hline$\sigma$ & $\mathrm{t}$ & 0 & 20000 & 80000 \\
\hline$C P$ & $€ /$ unit & 350 & 420 & 730 \\
\hline$C A P$ & $€$ /unit & \multicolumn{3}{|c|}{380} \\
\hline$C R$ & - & $23 \%$ & $23 \%$ & $23 \%$ \\
\hline
\end{tabular}

In the inferior situation, due to immature low carbon development, the simulation results show that carbon evolution of intangible assets is not harmonious. It mainly takes carbon sinks as the way of carbon compliance. This is the embodiment of the imbalance of enterprise resource configuration, as shown in Fig. 2. Under the medium situation, the evolution of enterprise carbon intangible assets has a significant improvement over the inferior circumstance. Namely, it demonstrates more coordination across various carbon intangible assets. This can be seen from Fig. 3.

Enterprises in the advantaged situation own more advanced technologies and certain brand accumulation foundation. Therefore, the contribution value created by carbon intangible assets is more significant. Enterprises can allocate resources more scientifically and rationally, in order to accumulate the assets. The characteristics of enterprises overall evolution are more prominent in the advantaged situations. Although there is no accumulation of the development of the assets in the beginning, the improvement space is large. When the enterprise makes corresponding adjustments that meet the requirements of low carbon development strategy, the value contribution of carbon intangible asset Agents shows obvious emergent property in the evolution process. The low carbon technology, low carbon brand and carbon value chain system are most prominent. This is illustrated in Fig. 4.

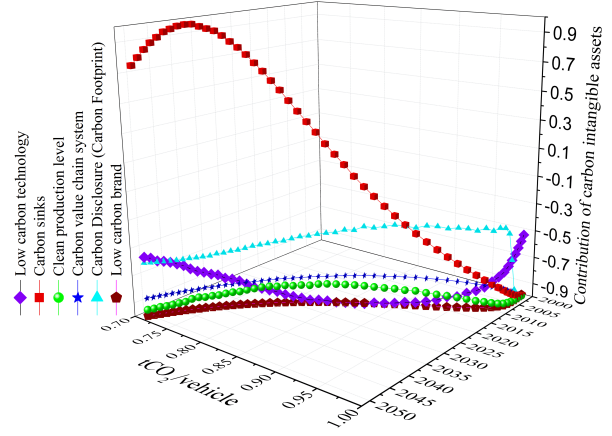

Figure 2. The evolution of carbon intangible assets in the inferior situation

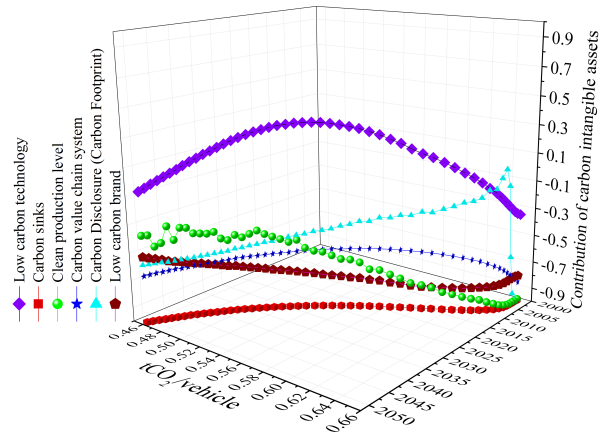

Figure 3. The evolution of carbon intangible assets in the medium situation

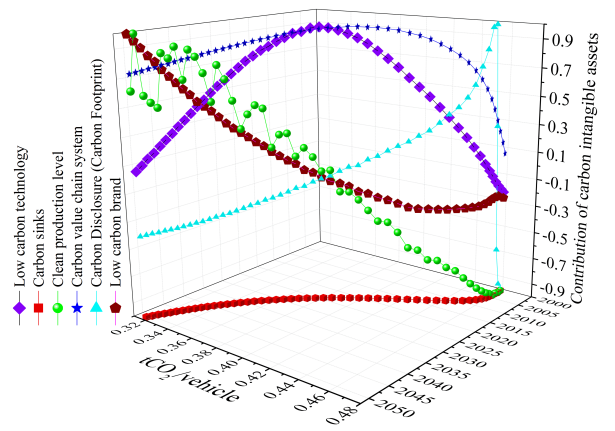

Figure 4. The evolution of carbon intangible assets in the advantaged situation

In the process of diffusion, advantaged enterprises can make profits from low carbon products and transfer low carbon technologies. The disadvantaged enterprises either pay the price to introduce low carbon technology to obtain the long-term development opportunities, or stay on the sidelines and may be eliminated. From Fig. 5, we can see that the simulation results of the three scenarios demonstrate the diffusion phenomenon of low carbon technologies. Enterprises under the inferior situation know their low carbon development level and understand that their low 
Zeng, Yin \& Li, Shuliang \& Deng, Liangru. "The measurement, mathematical and logical modeling, and agent-based simulation of carbon intangible assets with embedded strategies". Proceedings of the 2nd International Conference on Knowledge Engineering and Applications (ICKEA2017), pp.70-74. 21 st to 23rd Oct 2017, Imperial College London, UK. IEEE. ISBN 978-1-5386-2149-3 \& ISBN 978-1-5386-2148-6/17

carbon technologies do not have any advantages. They are faced with a great risk that the carbon intangible assets will not accumulate. They will lose the opportunity for further development, because the core assets, the emergence of low carbon technology and low carbon brand requires a much longer time to build up. Enterprises in advantaged and medium situations already take a leading role or have certain advantages to develop new energy automobiles. The simulation demonstrates the development trend between the two that advantaged enterprises choose to reduce the production of traditional fuel car models significantly faster and will be fully realized by 2025 , and even a small number of conventional fuel car models are still just out of consideration for some consumers. Enterprises under the medium condition implement both strategies to maintain the full research and development of new energy vehicles, and slow down the production of traditional energy car models. This can avoid entering the new energy car market but losing sight of the conventional market. The simulation results suggest that enterprises in disadvantaged situation fail to launch new energy vehicles, although some enterprises have been doing their best on research and development. Their business still reply on the traditional fuel car models. However, the market space for the old models will be very limited in the future. The simulation results reflect well on the law to develop low carbon technologies for automobile enterprises in the three scenarios. The strong one is going to be stronger.

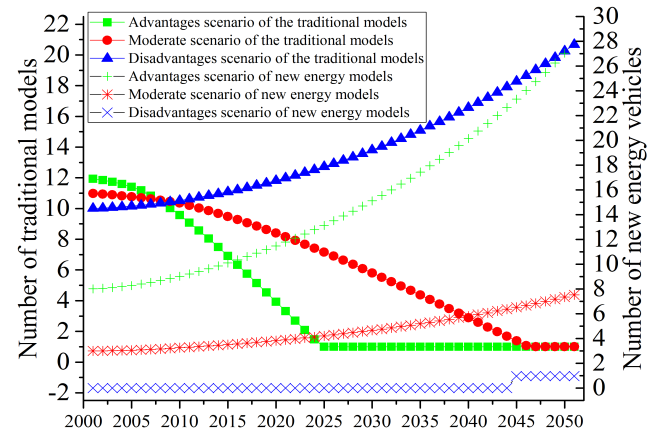

Figure 5. Comparison of the evolution of traditional fuel car models with new energy models in the three scenarios

\section{CONCLUSION}

The value model of the carbon intangible assets provides theoretical reference and foundation for automobile enterprises to make quantitative analysis of its low carbon advantages. This make it possible to accurately measure the assets obtained through the low carbon development. And thus help enterprises identity and investigate their advantages and disadvantages. Furthermore, the software agent simulation model for the dynamic evolution of carbon intangible asses based upon the value model and interactive mechanism can realistically support the low carbon development activity and help formulate management strategies for automobile enterprises. It can also assist with managers on analyzing and predicting the development laws and emergent properties of carbon intangible assets based upon historical and current data and information.

\section{ACKNOWLEDGMENT}

This study is supported by Chengdu (China) Soft Science Project - Research on the Mechanism Innovation of National Carbon Market Capacity Development (Chengdu) Center under the Perspective of Enterprise Carbon Assets (No.2016RK00-00076-ZF), the National Natural Science Foundation of China under grant No. 71472158. Sichuan 100-Talent Scheme grant (China), Southwest Jiaotong University (China) PhD scholarship, and the staff research allowances of the University of Westminste (UK).

\section{REFERENCES}

[1] F. Berkhout, A. Smith, "Carbon Flows Between the Eu and Eastern Europe: Baselines, Scenarios and Policy Options," International Environmental Agreements, vol. 3, Mar. 2003, pp. 199-219.

[2] E. Günther. Emissions Trading and Business, Physica-Verlag Heidelberg, 2006, pp. 219-239.

[3] S. Lewandowski. Corporate Carbon and Climate Accounting, Springer International Publishing, 2015, pp. 193-215.

[4] I. Gallego-alvarez, J. Martínez-ferrero, B. Cuadrado-ballesteros, "Accounting Treatment for Carbon Emission Rights," Systems, vol. 1(12), Apr. 2016, pp. 1-15.

[5] J. Munro. "Trade in Carbon Units as a Financial Service Under International Trade Law: Recent Developments, Future Challenges" ,Carbon \& Climate Law Review: CCLR, vol. 2, Aug. 2014, pp. 106114.

[6] R. Orth, R. Scheumann, M. Galeitzke,K. Wolf, H. Kohl,M Finkbeiner, "Sustainable Corporate Development Measured By Intangible and Tangible Resources as Well as Targeted By Safeguard Subjects," Procedia Cirp, vol. 26, Mar. 2015, pp. 630-634.

[7] Y. Fujii. Handbook of Climate Change Mitigation and Adaptation,Springer International Publishing, 2017, pp. 527-554.

[8] Y. Zeng, S. Li. "Agent-based Simulation of the Evolution of Enterprise Carbon Assets," Robots \& Intelligent System (ICRIS), 2016 International Conference on. IEEE,pp.81-84, doi: 10.1109/ICRIS.2016.16

[9] L. Han,Y. Liu, Q. Lin,G. Huang, "Valuing Carbon Assets for Hightech with Application to the Wind Energy Industry," Energy Policy, vol. 87, Dec. 2015, pp. 347-358.

[10] Z. Wang, C. Wang, "How Carbon Offsetting Scheme Impacts the Duopoly Output in Production and Abatement: Analysis in the Context of Carbon Cap-and-trade," Journal of Cleaner Production, vol. 103, Sep. 2015, pp. 715-723.

[11] V. Bosetti,C. Carraro, M. Galeotti. Stabilisation Targets, Technical Change and the Macroeconomic Costs of Climate Change Control, Feem Working Paper, 2006.

[12] S. Haque, M.A. Islam, "Stakeholder Pressures on Corporate Climate Change-related Accountability and Disclosures: Australian Evidence," Business and Politics, vol. 17, Aug. 2015, pp. 355-390.

[13] F. Völckner,H. Sattler, "Drivers of Brand Extension Success", Journal of Marketing, vol. 70, Apr. 2006, pp. 18-34.

[14] S. F. Railsback, S .L. Lytinen, S.K. Jackson, "Agent-based Simulation Platforms: Review and Development Recommendations", Simulation, vol. 82, Sep. 2006, pp. 609-623.

[15] X. Liu,Y. Fan, C. Li, "Carbon Pricing for Low Carbon Technology Diffusion: a Survey Analysis of China's Cement Industry," Energy, vol. 106, Jul. 2016, pp. 73-86. 